\title{
Mitotic Activity
}

National Cancer Institute

\section{Source}

National Cancer Institute. Mitotic Activity. NCI Thesaurus. Code C35959.

Having to do with the presence of dividing (proliferating) cells. Cancerous tissue generally has more mitotic activity than normal tissues. 